\title{
Two-meson cloud contribution to the baryon antidecuplet self-energy
}

\author{
T. Hyodo ${ }^{\mathrm{a}}$, A. Hosaka ${ }^{\mathrm{a}}$, F.J. Llanes-Estrada ${ }^{\mathrm{b}}$, E. Oset ${ }^{\mathrm{c}}$, J. R. Peláez ${ }^{\mathrm{d}}$ and \\ M. J. Vicente Vacas $^{\mathrm{c}}$,

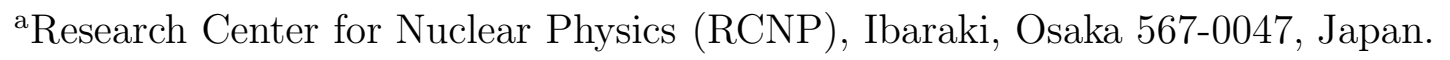 \\ bUniversidad Complutense de Madrid, Depto. Física Teórica I, 28040 Madrid, Spain. \\ ${ }^{\mathrm{c}}$ Departamento de Física Teórica and IFIC, Centro Mixto Universidad de Valencia-CSIC, \\ Institutos de Investigación de Paterna, Aptd. 22085, 46071 Valencia, Spain. \\ ${ }^{\mathrm{d} U n i v e r s i d a d ~ C o m p l u t e n s e ~ d e ~ M a d r i d, ~ D e p t o . ~ F i ́ s i c a ~ T e o ́ r i c a ~ I I, ~} 28040$ Madrid, Spain.
}

We study the self-energy of the $S U(3)$ antidecuplet coming from two-meson virtual clouds. Assuming that the exotic $\Theta^{+}$belongs to an antidecuplet representation with $N(1710)$ as nucleon partner, we derive effective Lagrangians that describe the decay of $N(1710)$ into $N \pi \pi$ with two pions in $s$ - or $p$-wave. It is found that the self-energies for all members of the antidecuplet are attractive, and the larger strangeness particle is more bound. From two-meson cloud, we obtain about $20 \%$ of the empirical mass splitting between states with different strangeness.

\section{Introduction}

In recent years, the study of the exotic pentaquark baryons has been one of the most exciting fields. Evidence of exotic $\Theta^{+}$was reported in Ref. [1] and a signal of another exotic state $\Xi^{--}$was subsequently observed [2], though the experimental confirmation of the latter is somehow controversial. In hadron spectroscopy, the Gell-Mann-Okubo[GMO] formula has been successfully applied to describe the mass splitting of particles within an $S U(3)$ multiplet. The smallest multiplet containing $\Theta^{+}$and $\Xi^{--}$is the antidecuplet $(\overline{\mathbf{1 0}})$, and equal mass splitting is obtained from the GMO rule. Here we would like to study the two-meson cloud effect to the baryon antidecuplet, which would contribute to mass splitting in addition to the GMO formula.

The study of the two-meson cloud effect is motivated by the attempts of constructing the $\Theta^{+}$as a $K \pi N$ bound state [ 3, 4, 5, 6], in which an attractive interaction is found for the $K \pi N$ states of $J^{P}=1 / 2^{+}$. Although the found attraction is not enough to bind the three body system, such a configuration is naturally expected in the $\Theta^{+}$structure, by observing that the $K \pi N$ mass is only $30 \mathrm{MeV}$ above the $\Theta^{+}$state.

In the present work, assuming that the $N(1710)$ has a large antidecuplet component, we construct flavor $S U(3)$ effective interaction Lagrangians, which account for the decay modes of the $N(1710)$ into $N \pi \pi$. Using these interaction Lagrangians, we calculate the self-energy of the antidecuplet. Details of the study can be found in Ref. [ 7]. 


\section{Formulation}

The interaction Lagrangians are constrained to be $S U(3)$ symmetric. The process we are considering is $\mathbf{8}_{M}+\mathbf{8}_{M}+\mathbf{8}_{B} \rightarrow \overline{\mathbf{1 0}}_{P}$, where we denote the octet baryon, meson and antidecuplet baryon as $\mathbf{8}_{B}, \mathbf{8}_{M}$ and $\overline{\mathbf{1 0}}$, respectively. In order to construct a singlet from the product of $\mathbf{8}_{M}, \mathbf{8}_{M}, \mathbf{8}_{B}$, and $\overline{\mathbf{1 0}} \bar{P}_{P}$, there are four combinations, in which two $\mathbf{8}_{M}$ mesons are combined into $\mathbf{8}_{M M}^{s}, \mathbf{8}_{M M}^{a}, \mathbf{1 0}_{M M}$ and $\mathbf{2 7 _ { M M }}$. However, two of them $\left(\mathbf{8}_{M M}^{a}\right.$, $\mathbf{1 0}_{M M}$ ) are identically zero, due to additional symmetry under exchange of two mesons. Hence, without using derivatives, we can construct the following effective Lagrangians:

$$
\begin{aligned}
\mathcal{L}^{8 s} & =\frac{g^{8 s}}{2 f} \bar{P}_{i j k} \epsilon^{l m k} \phi_{l}{ }^{a} \phi_{a}{ }^{i} B_{m}{ }^{j}+\text { h.c. }, \\
\mathcal{L}^{27} & =\frac{g^{27}}{2 f}\left[4 \bar{P}_{i j k} \epsilon^{l b k} \phi_{l}{ }^{i} \phi_{a}{ }^{j} B_{b}{ }^{a}-\frac{4}{5} \bar{P}_{i j k} \epsilon^{l b k} \phi_{l}{ }^{a} \phi_{a}{ }^{j} B_{b}{ }^{i}\right]+\text { h.c. },
\end{aligned}
$$

where $P, B$ and $\phi$ are the baryon antidecuplet, baryon octet and meson octet fields, respectively. We have included a factor $1 / 2 f$ to make $g^{8 s}$ and $g^{27}$ dimensionless $(f=93$ $\mathrm{MeV}$ is the pion decay constant). In the low momentum expansion, the above Lagrangians are the two lowest ones. In practice, however, they are not sufficient to account for the experimental decay of $N(1710)$ into $N \pi \pi$ (p-wave). In order to reproduce such decay mode, we introduce a Lagrangian with one derivative:

$$
\mathcal{L}^{8 a}=i \frac{g^{8 a}}{4 f^{2}} \bar{P}_{i j k} \epsilon^{l m k} \gamma^{\mu}\left(\partial_{\mu} \phi_{l}^{a} \phi_{a}^{i}-\phi_{l}^{a} \partial_{\mu} \phi_{a}^{i}\right) B_{m}^{j}+\text { h.c. . }
$$

We will address other possible interaction Lagrangians [7] later on.

The antidecuplet self-energies are given by

$$
\Sigma_{P}^{(j)}\left(p^{0}\right)=\sum_{B, m_{1}, m_{2}}\left(F^{(j)} C_{P, B, m_{1}, m_{2}}^{(j)}\right) I^{(j)}\left(p^{0} ; B, m_{1}, m_{2}\right)\left(F^{(j)} C_{P, B, m_{1}, m_{2}}^{(j)}\right),
$$

where the index $j$ labels the interaction Lagrangians, $p^{0}$ is the energy of the antidecu-

plet baryon, $F^{(j)}$ are coupling constants appearing in the Lagrangian, and $C_{P, B, m_{1}, m_{2}}^{(j)}$ are $S U(3)$ coefficients which are compiled in the Appendix of Ref. [7]. The function $I^{(j)}\left(p^{0} ; B, m_{1}, m_{2}\right)$ is the two-loop integral with two mesons and one baryon (Fig. 1, left):

$$
\begin{aligned}
& I^{(j)}\left(p^{0} ; B, m_{1}, m_{2}\right) \\
= & -\int \frac{d^{4} k}{(2 \pi)^{4}} \int \frac{d^{4} q}{(2 \pi)^{4}}\left|t^{(j)}\right|^{2} \frac{1}{k^{2}-m_{1}^{2}+i \epsilon} \frac{1}{q^{2}-m_{2}^{2}+i \epsilon} \frac{M}{E} \frac{1}{p^{0}-k^{0}-q^{0}-E+i \epsilon},
\end{aligned}
$$

where $t^{(j)}$ are the amplitudes derived from the Lagrangian $j, M$ and $m_{i}$ are the masses of a baryon and mesons, $E$ is the energy of the intermediate baryon. The real part of this integral is cut off with a three momentum $\Lambda$ in the range $700-800 \mathrm{MeV}$. The imaginary part of the diagram provides the decay width.

It is known that $N(1710) \rightarrow N \pi \pi(p$-wave) occurs through the $N \rho$ decay, therefore, we improve the contact interaction of the $\mathcal{L}^{8 a}$ to account for the vector meson propagator (Fig. 1, right), including the factor $m_{v}^{2} /\left[(q+k)^{2}-m_{v}^{2}\right]$ in each $P \rightarrow B M M$ vertex. 

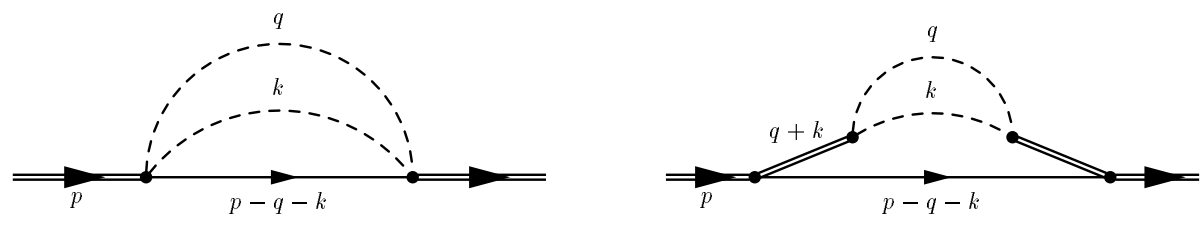

Figure 1. Diagrams for self-energy of baryon antidecuplet due to two-meson cloud. Right : inclusion of vector meson propagator.
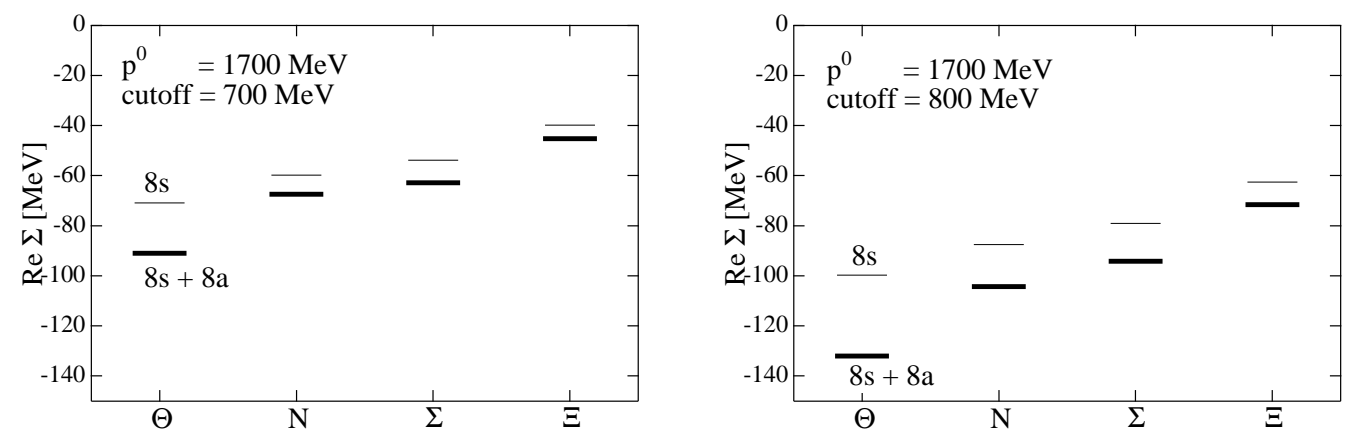

Figure 2. Mass shifts of baryon antidecuplet $\left(\operatorname{Re} \Sigma_{P}\right)$ with $p^{0}=1700 \mathrm{MeV}$.

\section{Numerical results}

Here we show the results with $\mathcal{L}^{8 s}$ and $\mathcal{L}^{8 a} \cdot \mathcal{L}^{27}$ and other possible Lagrangians will be addressed later. The parameters $g^{8 s}$ and $g^{8 a}$ are fixed so as to to obtain the partial decay widths of the $N(1710)$ to $N \pi \pi(s$-wave, isoscalar) and $N \rho \rightarrow N \pi \pi(p$-wave, isovector) respectively. The central values in the PDG [8] are 25 and $15 \mathrm{MeV}$, which correspond to $g^{8 s}=1.9$ and $g^{8 a}=0.32$, respectively. We take an average value of $p^{0}=1700 \mathrm{MeV}$ as input. The qualitative trend of the result does not depend on the $p^{0}$, but the magnitude of the self-energy is changed [ 7].

In Fig. 2 we show the real parts of the self-energies for the contributions from $\mathcal{L}^{8 s}$ and total contributions of $\mathcal{L}^{8 a}$ and $\mathcal{L}^{8 s}$, with cutoffs 700 and $800 \mathrm{MeV}$. We see that all the self-energies are attractive, and that the interaction is more attractive the larger the strangeness. $\mathcal{L}^{8 s}$ provides more binding than $\mathcal{L}^{8 a}$ for the same cutoff. The splitting between the $\Theta_{\overline{10}}$ and $\Xi_{\overline{10}}$ states is about $45-60 \mathrm{MeV}$ depending on the cutoff. Since the experimental splitting of the $\Theta(1540)$ and $\Xi(1860)$ is $320 \mathrm{MeV}$, the two-meson cloud provides $20 \%$ of the total splitting. This should be compared to $60 \%$ naturally provided by the mass of the constituent strange quarks, which would leave about $20 \%$ more for the effects of quark correlations.

The partial decay widths are shown in Table 1. We have taken the observed masses $M_{N_{\overline{10}}}=1710, M_{\Sigma_{\overline{10}}}=1770$ and $M_{\Xi_{\overline{10}}}=1860 \mathrm{MeV}$ as $p^{0}$, because the phase space is essential for the decay width. We can see that the widths are not very large for all channels. Indeed, $\Sigma(1770)$ and $\Xi(1860)$ would have widths for three body channels of about 24 and $2 \mathrm{MeV}$, which are compatible with the experimental total width of about 70 and $18 \mathrm{MeV}$, respectively [2, 8]. Detailed information of the partial decay widths of 
Table 1

Partial decay widths for the allowed channels and total width for any $B M M$ channel, at the masses of the antidecuplet members. All values are listed in units of $\mathrm{MeV}$.

\begin{tabular}{llll}
\hline Decay widths $[\mathrm{MeV}]$ & $\Gamma^{(8 s)}$ & $\Gamma^{(8 a)}$ & $\Gamma_{B M M}^{\text {tot }}$ \\
\hline$N(1710) \rightarrow N \pi \pi$ (inputs) & 25 & 15 & 40 \\
$N(1710) \rightarrow N \eta \pi$ & 0.58 & - & \\
$\Sigma(1770) \rightarrow N \bar{K} \pi$ & 4.7 & 6.0 & 24 \\
$\Sigma(1770) \rightarrow \Sigma \pi \pi$ & 10 & 0.62 & \\
$\Sigma(1770) \rightarrow \Lambda \pi \pi$ & - & 2.9 & \\
$\Xi(1860) \rightarrow \Sigma \bar{K} \pi$ & 0.57 & 0.46 & 2.1 \\
$\Xi(1860) \rightarrow \Xi \pi \pi$ & - & 1.1 & \\
\hline
\end{tabular}

three body channels will give us more understanding of the interaction Lagrangian.

In Ref [ 1], apart from the above Lagrangians and a $\mathcal{L}^{27}$ term, we also considered the leading order Lagrangians in a chiral expansion as dictated by QCD. There are two terms: one chirally symmetric and a mass term. The latter has to be tiny since it violates $\mathrm{SU}(3)$. The former gives similar results to $\mathcal{L}^{8 s}$. The role of chiral symmetry is thus small and the use of $\mathcal{L}^{8 s}$ is justified.

\section{Summary}

We study the self-energy of the baryon antidecuplet due to the two-meson cloud. The assumptions made throughout the paper and the uncertainties in the experimental input make the nature of our analysis qualitative. However, in all different cases studied, the two-meson cloud mechanism leads to the following conclusions: An attractive self-energy is obtained for all members of the antidecuplet. The two-meson cloud contributes to the mass splitting between antidecuplet members about $20 \%$ of the empirical one. These observations are consistent with the previous attempts to describe the $\Theta^{+}$as a $K \pi N$ state [ 3, 4, 5, 6], and the magnitude of $20 \%$ is also in agreement quantitatively with the strength of attraction [ 5]. The role played by the two-meson cloud is therefore of relevance for a precise understanding of the nature of the $\Theta^{+}$and the antidecuplet.

\section{REFERENCES}

1. LEPS, T. Nakano et al., Phys. Rev. Lett. 91, 012002 (2003).

2. NA49, C. Alt et al., Phys. Rev. Lett. 92, 042003 (2004).

3. P. Bicudo and G. M. Marques, Phys. Rev. D69, 011503 (2004).

4. T. Kishimoto and T. Sato, hep-ex/0312003.

5. F. J. Llanes-Estrada, E. Oset, and V. Mateu, Phys. Rev. C69, 055203 (2004).

6. P. Bicudo, hep-ph/0403146.

7. A. Hosaka, T. Hyodo, F.J. Llanes-Estrada, E. Oset, J. R. Peláez, and M. J. Vicente Vacas, hep-ph/0411311.

8. Particle Data Group, S. Eidelman et al., Phys. Lett. B592, 1 (2004). 